\title{
The Effectiveness of Routine Cranial Computerized Tomography in the Evaluation of Facial Bone Fractures
}

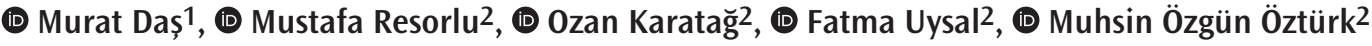 \\ 1Department of Emergency Department, Çanakkale Onsekiz Mart University Faculty of Medicine, Çanakkale, Turkey \\ 2Department of Radiology, Çanakkale Onsekiz Mart University Faculty of Medicine, Çanakkale, Turkey
}

\begin{abstract}
Aim: This study aimed to investigate the ability of routine cranial computed tomography (CT) to detect facial bone fractures, taking the maxillofacial, orbital, and temporal bones $\mathrm{CT}$ as reference.

Materials and Methods: Patients who presented to the emergency department with head trauma and undergoing cranial CT were included in the study. Cases in the study group were also assessed using at least one of maxillofacial, orbital, or temporal CT.

Results: Fracture was present in 155 patients who were examined by maxillofacial, orbital, or temporal CT. Moreover, 59 patients had a single fracture and 96 had more than one fracture. Fractures were determined completely and accurately in 71 patients using cranial CT. Cranial CT successfully provided the fracture diagnosis in 48 of 96 patients with multiple fractures, but all fracture lines in these patients were not shown. Eleven patients were reported as having false-positive results. The effectiveness of cranial CT was presented as a sensitivity of $45.8 \%$, specificity of $93.1 \%$, positive predictive value of $86.6 \%$, negative predictive value of $63.8 \%$, and kappa value of 0.39 . Cranial CT identified 11 of 21 temporal, 33 of 50 nasal, 27 of 35 zygomatic, 3 of 4 occipital, 8 of 17 ethmoid, and 19 of 23 frontal bone fractures.

Conclusion: CT assists in the detection of small, non-displaced fractures at the temporal, maxillofacial, and orbital bones owing to its advantages such as having thin slice thickness, use of a bone algorithm, and ability to reformat images.
\end{abstract}

Keywords: Emergency department, fractures, maxillofacial injuries, multidetector computed tomography

\section{Introduction}

Trauma is a significant public health problem and one of the main causes of death in individuals under 40 , in particular. Cerebral hemorrhage is significant in patients with general body trauma in terms of both its prevalence and fatal outcomes. The etiology of head trauma includes traffic accidents, accidents at work, violence, sports injuries and falls (1). Moreover, the prevalence of etiological factors varies depending on societies and sociocultural differences. For example, motor vehicle accidents predominate in developing countries and violence in developed countries $(1,2)$. Cerebral hemorrhage and accompanying fractures in facial bones are common in these patients.
The priority is to prevent mortality due to cerebral injury, function impairment due to facial injuries and cosmetic problems. Mortality may rarely be seen and is primarily associated with hemorrhaging, foreign bodies and airway obstruction (3). Cranial computed tomography (CT) and, more rarely, magnetic resonance imaging are used to assess cranial trauma. Facial osseous structures, such as the orbital, temporal and maxillofacial bones, can also be assessed by cranial CT. However, cranial CT may be insufficient due to factors such as slice thickness, imaging angle and imaging area. For this reason, maxillofacial, orbital and temporal bone CT images are frequently employed. In this study, we investigated the capacity of cranial CT to identify facial bone fractures by using maxillofacial, orbital and temporal bone CT as a reference. 


\section{Materials and Methods}

The study population was selected from among patients presenting to the emergency department due to trauma and was assessed using cranial CT. Additionally, 314 patients assessed with at least one of a temporal bone, orbital or maxillofacial CT were enrolled in the study. Insufficient image quality was regarded as an exclusion criterion. All patients were evaluated by two radiologists, the first of whom reported orbital, temporal bone and maxillofacial CTs, while the second, blinded to those results, reported cranial CT.

The temporal, orbital and maxillofacial CT results were adopted as a reference, and the ability of cranial CT to detect bone fractures was evaluated. Approval was granted by our hospital's ethical committee. Furthermore, statistical analysis was performed using SPSS 19.0 software. Descriptive data were expressed as mean, standard deviation, minimum, maximum, percentage and frequency values. Comparisons between groups were performed using the Kruskal-Wallis and Mann-Whitney $\mathrm{U}$ tests for constant variables and the chi-square test for categoric variables. Moreover, the Cohen kappa statistical method was used to assess agreement between radiological methods.

\section{Imaging Protocol}

All patients were evaluated with a 4-multidetector CT scanner (Toshiba ${ }^{\circledR}$ Asteion TSX-021B) without intravenous or oral contrast media. CT imaging was performed according to the routine protocol employed in our hospital, with the removal of metal objects capable of causing artifacts, such as hair clips, earrings, and so on. The imaging field at maxillofacial CT comprised the mandible in the inferior aspect and the frontal sinus in the superior. The imaging protocol consisted of a slice thickness of $2 \mathrm{~mm}, 150 \mathrm{~mA}$ and $120 \mathrm{kV}$. Thin-slice axial source images and coronal-sagittal multiplanar reformation images were evaluated in the bone and soft tissue algorithm.

Axial images at orbital CT were obtained parallel to the orbitomeatal line. The imaging protocol consisted of a slice thickness of $1 \mathrm{~mm}, 80 \mathrm{~mA}$ and $120 \mathrm{kV}$. Cranial CT was performed from the foramen magnum as far as the vertex at a slice thickness of $8 \mathrm{~mm}$ in the supratentorial region and $4 \mathrm{~mm}$ in the infratentorial region, and at $200 \mathrm{~mA}$ and $120 \mathrm{kV}$.

Temporal bone CT was performed between the beginning of the mastoid air cells and the superior margin of the petrous bone, parallel to the infraorbital line, and in the bone algorithm. Parameters of a slice thickness of $1 \mathrm{~mm}, 120 \mathrm{kV}$ and $150 \mathrm{~mA}$ were employed. No contrast media was used in any case during imaging.

\section{Results}

The mean age of the 314 patients in the study, consisting of 91 women (29\%) and 223 men (71\%), was 41.15-21.19 years. All patients underwent at least one of a maxillofacial, orbital or temporal bone $\mathrm{CT}$ in addition to cranial $\mathrm{CT}$. Fractures were determined in 155 patients (49.4\%) upon CT examination of the facial bones, while 159 (50.6\%) patients were normal. A single fracture was present in 59 of the patients with fractures, and multiple fractures were present in 96. Additionally, 71 patients with fractures were reported fully and accurately with cranial $\mathrm{CT}$, while 36 were misdiagnosed as normal. While fractures were determined in 48 of the 96 multiple-fracture patients, not all fracture lines could be displayed. False positivity was reported in 11 patients. The ability of cranial CT to reveal the presence of fractures and reveal all fractures accurately and completely is as follows: sensitivity, $45.8 \%$; specificity, $93.1 \%$; positive predictive value (PPV), 86.6\%; negative predictive value (NPV), 63.8\%; and kappa value, 0.39. The effectiveness of cranial CT for facial fractures is presented in Table 1.

In terms of location, fractures in the temporal bone were present in 21 patients. This was correctly identified in 11 patients upon undergoing cranial $\mathrm{CT}$, while false negativity was present in 10, and two cases were incorrectly reported as having fractures (Figure 1)

Thirty-three of the 50 patients with nasal bone fractures and 27 of the 35 with zygomatic fractures were reported correctly with cranial CT. False positivity in the zygomatic and nasal bones was present in one patient each at cranial CT. Fractures in the maxilla was present in 42 patients, 27 of whom were reported correctly, while fractures could not be shown in 15 , and false positivity was present in three patients. Thirty patients had orbital fractures, which were displayed in 18 of these patients with cranial CT, while false positivity was present in two patients. Five of the 14 patients with sphenoid fractures were reported correctly, while false negativity was present in nine and false positivity in one. Three of the four patients with occipital fractures (Figure 2), eight

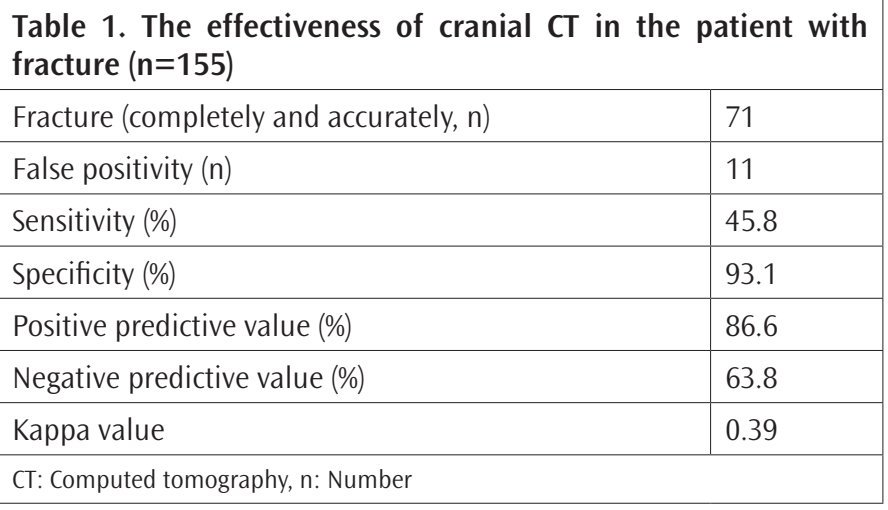


of the 17 with ethmoid fractures, and 19 of the 23 with frontal fractures were reported correctly. The sensitivity, specificity, PPV and NPV values for cranial CT fracture locations are presented in Table 2.
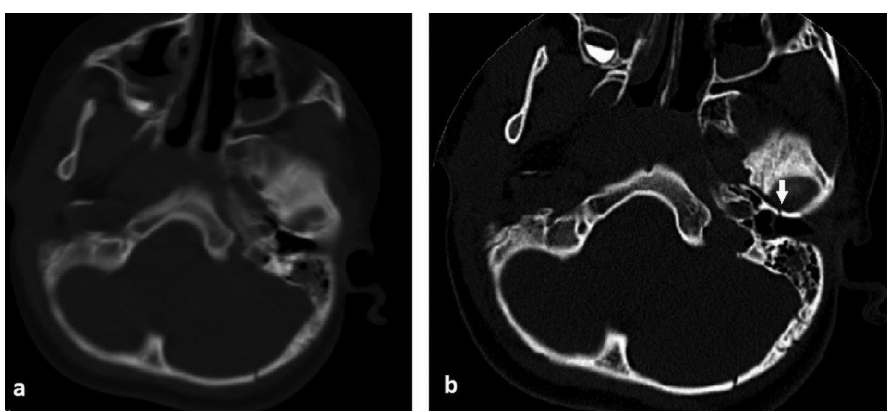

Figure 1. Axial non-contrast cranial (a) and temporal bone CT (b). Axial high-resolution multi-detector $C T$ image of the temporal bone reveals a fracture on the anterior wall of the external auditory canal

CT: Computed tomography
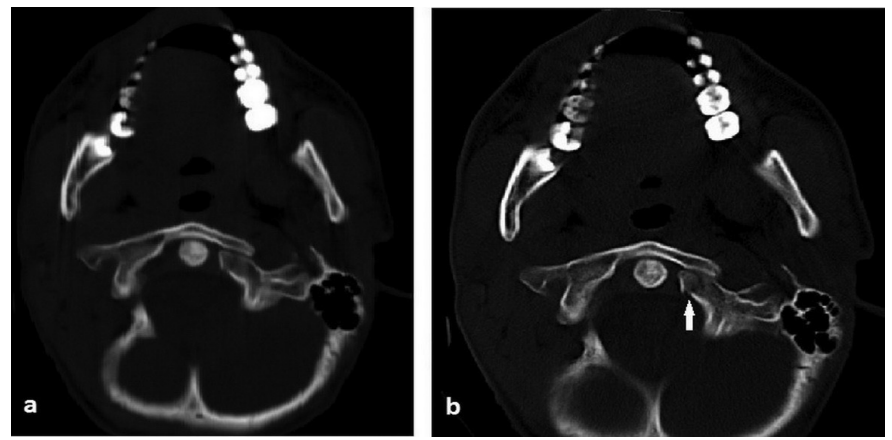

Figure 2. Cranial (a) and maxillofascial (b) axial CT images from skull base. A fracture line is present on the left occipital condyle

$\mathrm{CT}$ : Computed tomography

\section{Discussion}

Facial trauma and fractures are significant health problems resulting in economic losses due to their high incidence. Facial fractures are not often life-threatening, though complications include impairment of functions, such as sight, hearing and smell, and esthetic problems (3,4). The incidence of complications increases in high-energy trauma and complicated fractures. One study reported life-threatening injuries such as hemorrhagic shock or airway obstruction in facial trauma with an incidence of $6.2 \%(5)$.

The etiology of trauma varies among countries and even in different sociocultural regions within the same country (1). The mean age in the study by Shah et al. (6) was 33.7 years. Meanwhile, Sohns et al. (7) observed sex distributions of $36 \%$ in females and $64 \%$ in males. The mean age in our study was 41.15 years, with a sex distribution of 91 females (29\%) and 223 (71\%) males, which aligns with the previous literature. Factors such as the use of fast vehicles, dangerous sports activities and physical violence affect the variation in terms of age and sex. In addition, given that the facial bones and paranasal sinuses are not fully developed in children, the small volume of their facial bones and the flexibility of the facial structures suggest that facial fractures are less common in children (8).

Since fractures were detected in only 155 of the 314 subjects who underwent facial CT in this study, this suggests that maxillofacial CT was requested unnecessarily in approximately half of the patients. However, fractures could be detected in only 119 of the 155 fracture patients using cranial $\mathrm{CT}$, and false positivity was diagnosed in 11 patients. While fractures were shown in 48 of the 96 patients with multifractures, not all fractures' lines were detected. Cranial CT exhibited $45.8 \%$ sensitivity, $93.1 \%$ specificity

Table 2. Fracture locations and the effectiveness of cranial CT

Cranial CT results

\begin{tabular}{|l|l|l|l|l|l|l|l|}
\hline Localization & Fracture $(\mathbf{n})$ & Fracture & Sensitivity (\%) & Specificity (\%) & PPV & NPV & Kappa \\
\hline Nasal & 50 & 33 & 66 & 99.6 & 97.1 & 93.9 & 0.75 \\
\hline Maxilla & 42 & 27 & 64.3 & 98.9 & 90.0 & 94.7 & 0.71 \\
\hline Zygomatic & 35 & 27 & 77.1 & 99.6 & 96.4 & 97.2 & 0.84 \\
\hline Orbita & 30 & 18 & 60.0 & 99.3 & 90.0 & 95.9 & 0.69 \\
\hline Frontal & 23 & 19 & 82.6 & 100 & 100 & 98.6 & 0.89 \\
\hline Temporal & 21 & 11 & 52.4 & 99.3 & 84.6 & 96.7 & 0.62 \\
\hline Ethmoid & 17 & 8 & 47.1 & 100 & 100 & 97.1 & 0.62 \\
\hline Mandible & 14 & 2 & 14.3 & 99.7 & 66.7 & 96.1 & 0.22 \\
\hline Sphenoid & 14 & 5 & 35.7 & 99.7 & 83.3 & 97.1 & 0.48 \\
\hline Occipital & 4 & 3 & 75 & 100 & 100 & 99.7 & 0.85 \\
\hline CT: Computed tomography, PPV: Positive predictive value, NPV: Negative predictive value, n: Number & & & \\
\hline
\end{tabular}


and a kappa value of 0.39 for facial fractures. Therefore, these results indicate that cranial $C T$ is insufficient when potential complications are considered. Additionally, because maxillofacial trauma is painful and the area of trauma is edematous, physical examination is insufficient and additional imaging is essential.

The most common sites of fracture in this study were, in descending order, the nasal bone, maxilla, zygomatic bone and orbital bone. Various previous studies have reported inconsistent findings in terms of fracture prevalence. For example, Sohns et al. (7) reported that fractures in the orbito-zygomatico area and maxilla fractures were more common, while Hwang and You (9) maintained that fractures were most prevalent in the nasal bone.

The nasal bone was also the most common site in our study. Deformities and nasal obstructions in nasal trauma can be determined through physical examination. Old fractures, vascular structures and suture lines yield false positive results upon radiography, while cartilaginous injuries yield false negative results (10). We were unable to identify 17 of the 50 patients with nasal fractures using cranial CT. The slice thickness affects the inability to identify nasal fractures, even though the nasal bone enters the imaging field.

Twenty-seven of the 42 patients with maxillary fractures and two of the 12 patients with mandibular fractures were reported correctly with cranial CT. The numbers of patients diagnosed with false positive fractures in these two sites at cranial CT were three and one, respectively. All the mandibular fractures could not be identified, and 8 of the 15 maxillary fractures were outside the cranial CT imaging field. Indirect findings, such as fluid inside the sinus or the presence of air contiguous to the sinus are suggestive of fractures, particularly in fractures affecting the maxillary sinus. Therefore, fractures involving the maxillary sinus are frequently accurately identified. Shah et al. (6) investigated the efficacy of three-dimensional computed tomography in the diagnosis of maxillofacial fractures. They compared their study findings with those of Baek et al. (11) and attributed the resulting discrepancies in the determination of undisplaced fractures to slice thickness (6). We were unable to identify seven of the maxillary fractures, though inside the cranial $\mathrm{CT}$ imaging field, these were undisplaced in character. This supports the assertion of Shah et al. (6).

Orbital trauma is frequently accompanied by multiple organ trauma. The second most common form of fractures, after blunt orbital fractures, is "blow-out" fractures. Compression fractures may be observed in the lamina papyracea that constitutes the medial wall of the orbit. Injuries to the dura occasionally accompany orbital roof fractures (12). Ophthalmological evaluation is limited by the presence of soft tissue swelling and pain. Radiological imaging is particularly important for diagnosis, and the gold standard imaging technique is orbital CT. A slice thickness $(0.5-1.25 \mathrm{~mm})$ and the presence of coronal and sagittal reformatted images in additional to axial plane images increase the diagnostic effectiveness. Kim et al. (13) compared orbital fractures in young and elderly patient groups. They reported that medial wall fractures were common in the non-elderly group, while lateral wall fractures were common in the elderly group. Eighteen of the 30 orbital fractures and 27 of the zygomatic fractures $(n=35)$ in our study were reported correctly at cranial CT. Coronal and sagittal reformatted images at orbital CT were particularly helpful in revealing fractures within the orbital floor and roof. Small compression fractures in the lamina papyracea that could not be revealed at cranial CT were identified with 1 $\mathrm{mm}$ thin slices at orbital CT.

Nineteen of the 23 patients with frontal fractures and three of the four patients with occipital fractures were shown correctly with cranial CT. The occipital fractures that could not be detected had nondisplaced character in the craniocervical junction. Similarly, non-detected frontal fractures were small and undisplaced. The temporal bone is one of the strongest bones, and fractures occur with severe trauma. Fractures are more common in cavities through which large vessels and nerves pass and in weak points containing mastoid cells. Fractures in the temporal bone are known to occur in $14-22 \%$ of patients with skull fractures $(14,15)$. Radiography and conventional tomography are insufficient for evaluation. Only half of the temporal bone fractures are reported to be capable of detection using cranial CT (16). Fractures can be detected to a significant extent with high resolution $\mathrm{CT}$ with a slice thickness of $1 \mathrm{~mm}$ and a bone algorithm. Additionally, transverse fractures can be identified more easily in sagittal images and longitudinal ones on coronal images (17). In our study, $52.3 \%$ of temporal fractures were identified correctly and fully with cranial CT, which aligns with the literature.

\section{Study Limitations}

This study had some limitations. In particular, the 4-detector tomography device used served as a leading limitation. We believe that that reliability could be increased in similar studies with new-generation multi-detector computed tomography devices.

\section{Conclusion}

Fractures in the body of the maxilla and mandible cannot be detected at cranial $\mathrm{CT}$, since they lie outside the imaging field. Temporal, maxillofacial and orbital CT offer many advantages in the detection of small undisplaced fractures, including a thin slice thickness, bone algorithm and reformatted image. 


\section{Ethics}

Ethics Committee Approval: The study was approved by the Rectorship of Çanakkale Onsekiz Mart University Deanship of the Faculty of Medicine Clinical Researches Ethics Committee (decision no: 2011-KAEK-27/2017-E.16899, date: 06.03.2017).

Informed Consent: Since this study was conducted retrospectively, informed consent was not obtained from the patients.

Peer-review: Externally peer-reviewed.

\section{Authorship Contributions}

Surgical and Medical Practices: M.D., M.R., O.K., F.U., M.Ö.Ö., Concept: M.D., Design: M.R., Data Collection or Processing: O.K., M.Ö.Ö., Analysis or Interpretation: M.D., Literature Search: M.R., Writing: M.D., M.R.

Conflict of Interest: No conflict of interest was declared by the authors.

Financial Disclosure: The authors declared that this study received no financial support.

\section{References}

1. Allareddy V, Allareddy V, Nalliah RP. Epidemiology of facial fracture injuries. J Oral Maxillofac Surg. 201;69:2613-8.

2. Aksoy $E$, Unlü $E$, Sensöz $O$. A retrospective study on epidemiology and treatment of maxillofacial fractures. J Craniofac Surg. 2002;13:772-5.

3. Kim JJ, Huoh K. Maxillofacial (midface) fractures. Neuroimaging Clin N Am. 2010;20:581-96

4. Ribeiro Ribeiro AL, da Silva Gillet LC, de Vasconcelos HG, de Castro Rodrigues L, de Jesus Viana Pinheiro J, de Melo Alves-Junior S. Facial Fractures: Large Epidemiologic Survey in Northern Brazil Reveals Some Unique Characteristics. J Oral Maxillofac Surg. 2016;74:2480.
5. Tung TC, Tseng WS, Chen CT, Lai JP, Chen YR. Acute life-threatening injuries in facial fracture patients: a review of 1,025 patients. J Trauma. 2000;49:420-4.

6. Shah S, Uppal SK, Mittal RK, Garg R, Saggar K, Dhawan R. Diagnostic tools in maxillofacial fractures: Is there really a need of three-dimensional computed tomography? Indian J Plast Surg. 2016;49:225-33.

7. Sohns JM, Staab W, Sohns C, Schwarz A, Streit U, Hosseini AS, et al. Current perspective of multidetector computed tomography (MDCT) in patients after midface and craniofacial trauma. Clin Imaging. 2013;37:728-33.

8. Shaikh ZS, Worrall SF. Epidemiology of facial trauma in a sample of patients aged 1-18 years. Injury. 2002;33:669-71.

9. Hwang K, You SH. Analysis of facial bone fractures: An 11-year study of 2,094 patients. Indian J Plast Surg. 2010;43:42-8.

10. Koh JH, Bhatti O, Mahmood A, Agar N. Traumatic nasal injuries in general practice. Aust Fam Physician. 2016;45:650-3.

11. Baek HJ, Kim DW, Ryu JH, Lee YJ. Identification of Nasal Bone Fractures on Conventional Radiography and Facial CT: Comparison of the Diagnostic Accuracy in Different Imaging Modalities and Analysis of Interobserver Reliability. Iran J Radiol. 2013;10:140-7.

12. Hopper RA, Salemy S, Sze RW. Diagnosis of midface fractures with CT: what the surgeon needs to know. Radiographics. 2006;26:783-93.

13. Kim YJ, Ahn S, Seo DW, Sohn CH, Lee HJ, Park IJ, et al. Patterns and injuries associated with orbital wall fractures in elderly patients who visited the emergency room: a retrospective case-control study. BMJ Open. 2016;6:e011110.

14. Brodie HA, Thompson TC. Management of complications from 820 temporal bone fractures. Am J Otol. 1997;18:188-97.

15. Nosan DK, Benecke JE Jr, Murr AH. Current perspective on temporal bone trauma. Otolaryngol Head Neck Surg. 1997;117:67-71.

16. Johnson DW, Hasso AN, Stewart CE 3rd, Thompson JR, Hinshaw DB Jr. Temporal bone trauma: high-resolution computed tomographic evaluation. Radiology. 1984;151:411-5.

17. Zayas JO, Feliciano YZ, Hadley CR, Gomez AA, Vidal JA. Temporal bone trauma and the role of multidetector $C T$ in the emergency department. Radiographics. 2011;31:1741-55. 